\title{
The homology of the stable nonorientable mapping class group
}

\author{
OSCAR RANDAL-WILLIAMS
}

\begin{abstract}
Combining results of Wahl, Galatius-Madsen-Tillmann-Weiss and Korkmaz, one can identify the homotopy type of the classifying space of the stable nonorientable mapping class group $\mathcal{N}_{\infty}$ (after plus-construction). At odd primes $p$, the $\mathbb{F}_{p}-$ homology coincides with that of $Q_{0}\left(\mathbb{H} \mathbb{P}_{+}^{\infty}\right)$, but at the prime 2 the result is less clear. We identify the $\mathbb{F}_{2}$-homology as a Hopf algebra in terms of the homology of well-known spaces. As an application we tabulate the integral stable homology of $\mathcal{N}_{\infty}$ in degrees up to six.

As in the oriented case, not all of these cohomology classes have a geometric interpretation. We determine a polynomial subalgebra of $H^{*}\left(\mathcal{N}_{\infty} ; \mathbf{F}_{2}\right)$ consisting of geometrically-defined characteristic classes.
\end{abstract}

57R20, 55P47; 55S12, 55T20

\section{Introduction}

The mapping class groups $\mathcal{N}_{g}$ of nonorientable surfaces are not as widely studied as their counterparts for oriented surfaces, but with Wahl's proof [16] of homological stability for these groups, one can apply the machinery of Madsen and Weiss [12] used to prove the Mumford conjecture or its more concise variant by Galatius, Madsen, Tillman and Weiss [8] to study their stable homology. Together these results show that the homology of $\mathcal{N}_{\infty}$ coincides with that of a component of an infinite loop space, $\Omega_{0}^{\infty} \mathbf{M T O}(2)$, which we define in Section 2.2.

Inspired by Galatius' calculation [7] of the homology of the stable oriented mapping class group $\Gamma_{\infty}$, we calculate the mod 2 homology of the stable nonorientable mapping class group $\mathcal{N}_{\infty}$. The odd-primary homology is much simpler: it coincides with that of $Q_{0}\left(\mathbb{H} \mathbb{P}_{+}^{\infty}\right)$ and is discussed in Section 5.1. Here $Q(X):=\operatorname{colim} \Omega^{n} \Sigma^{n} X$ denotes the free infinite loop space on the space $X$, the subscript 0 denotes the connected component of the constant loop and the subscript + denotes the addition of a disjoint basepoint. The homology $H_{*}\left(Q(X) ; \mathbb{F}_{p}\right)$ is completely known by Cohen, Lada and May [4] as a functor of $H_{*}\left(X ; \mathbb{F}_{p}\right)$. 
We adopt the convention that all (co)homology groups are with $\mathbb{F}_{2}$ coefficients unless otherwise stated. Our main theorems give a determination of the $\mathbb{F}_{2}$-homology of $\Omega_{0}^{\infty} \mathbf{M T O}(2)$ as a Hopf algebra:

Theorem A There is an exact sequence of Hopf algebras

$$
\mathbb{F}_{2} \longrightarrow H_{*}\left(\Omega_{0}^{\infty} \mathbf{M T O}(1)\right) \stackrel{\bar{\omega}_{*}}{\longrightarrow} H_{*}\left(Q_{0}\left(B O_{1+}\right)\right) \stackrel{\bar{\partial}_{*}}{\longrightarrow} H_{*}\left(Q_{0}\left(S^{0}\right)\right) \longrightarrow \mathbb{F}_{2}
$$

which is split as algebras.

Theorem B There is an exact sequence of Hopf algebras

$$
\mathbb{F}_{2} \longrightarrow H_{*}\left(\Omega_{0}^{\infty} \mathbf{M T O}(2)\right) \stackrel{\omega_{*}}{\longrightarrow} H_{*}\left(Q_{0}\left(B O_{2+}\right)\right) \stackrel{\partial_{*}}{\longrightarrow} H_{*}\left(\Omega_{0}^{\infty} \mathbf{M T O}(1)\right) \longrightarrow \mathbb{F}_{2}
$$

which is split as algebras.

We also give formulae for the maps $\bar{\partial}_{*}$ and $\partial_{*}$. Although these theorems determine the required homology groups, they do not do so in a very explicit manner. Thus we tabulate the first six (co)homology groups:

\begin{tabular}{lcccccc}
\hline Degree $i$ & 1 & 2 & 3 & 4 & 5 & 6 \\
\hline $\operatorname{Rank} Q H_{i}\left(\Omega_{0}^{\infty} \mathbf{M T O}(2) ; \mathbb{F}_{2}\right)$ & 1 & 2 & 3 & 3 & 5 & 6 \\
$\operatorname{Rank} H_{i}\left(\Omega_{0}^{\infty} \mathbf{M T O}(2) ; \mathbb{F}_{2}\right)$ & 1 & 3 & 6 & 12 & 23 & 45 \\
\hline$H_{i}\left(\Omega_{0}^{\infty} \mathbf{M T O}(2) ; \mathbb{Z}\right)$ & $\mathbb{Z}_{2}$ & $\mathbb{Z}_{2}^{2}$ & $\mathbb{Z}_{2}^{3} \oplus \mathbb{Z}_{12}$ & $\mathbb{Z}_{2}^{7} \oplus \mathbb{Z}$ & $\mathbb{Z}_{2}^{16}$ & $\mathbb{Z}_{2}^{29}$ \\
$H^{i}\left(\Omega_{0}^{\infty} \mathbf{M T O}(2) ; \mathbb{Z}\right)$ & 0 & $\mathbb{Z}_{2}$ & $\mathbb{Z}_{2}^{2}$ & $\mathbb{Z}_{2}^{3} \oplus \mathbb{Z}_{12} \oplus \mathbb{Z}$ & $\mathbb{Z}_{2}^{7}$ & $\mathbb{Z}_{2}^{16}$ \\
\hline
\end{tabular}

The integral homology can be calculated via the Bockstein spectral sequence. Only the primes 2 and 3 appear in this range: for the prime 3 the homology coincides with that of $Q\left(\mathbb{H} \mathbb{P}_{+}^{\infty}\right)$ and the Bockstein spectral sequence can be calculated from the known one for $\mathbb{H} \mathbb{P}^{\infty}$, which is degenerate from the first page. For the prime 2 we compare the Bockstein spectral sequence for $Q\left(\mathrm{BO}_{2+}\right)$ with the representing elements for classes belonging to $\Omega_{0}^{\infty} \mathbf{M T O}(2)$. We find that it degenerates at the $E^{3}$ page, giving the claimed homology groups.

We also study a family of mod 2 characteristic classes of surface bundles, $\chi_{i}$, defined as follows. For a surface bundle $F \longrightarrow E \stackrel{\pi}{\longrightarrow} B$ there is an associated vector bundle $V$ of first real cohomologies, with fibre $H^{1}\left(F_{b} ; \mathbb{R}\right)$ over $b \in B$. We can define

$$
\chi_{i}(E):=w_{i}(V) \in H^{i}\left(B ; \mathbb{F}_{2}\right)
$$


the $i$-th Stiefel-Whitney class of $V$. The main theorem is that these are stably independent for the nonorientable mapping class groups, so account for one indecomposable generator of the cohomology of $B \mathcal{N}_{\infty}$ in each dimension.

Theorem C In the nonorientable mapping class groups, the map

$$
\mathbb{F}_{2}\left[\chi_{1}, \chi_{2}, \chi_{3}, \ldots\right] \longrightarrow H^{*}\left(B \mathcal{N}_{g} ; \mathbb{F}_{2}\right)
$$

is an injection in the stable range $* \leq(g-3) / 4$.

Acknowledgements I gratefully acknowledge the support of an EPSRC Studentship, DTA grant number EP/P502667/1. I would like to thank the anonymous referee for their many helpful comments and suggestions.

\section{Recollections}

\subsection{Homological stability}

Write $N_{g, b}$ for the nonorientable surface consisting of the connected sum of $g$ copies of $\mathbb{R} \mathbb{P}^{2}$ with $b$ discs removed. We call $g$ the genus of the surface, and $b$ the number of boundary components, which we suppress in notation if it is 0 . The mapping class group of $N_{g, b}$ is the group of components of the topological group of self-diffeomorphisms that fix the boundary pointwise

$$
\mathcal{N}_{g, b}:=\pi_{0}\left(\operatorname{Diff}\left(N_{g, b}, \partial N_{g, b}\right)\right) .
$$

As long as $b \geq 1$ there are two stabilisation maps between these groups, obtained by gluing either a $N_{1,2}$ or a $N_{0,3}$ to a boundary component and extending diffeomorphisms by the identity to the new surface

$$
\begin{aligned}
\alpha: \mathcal{N}_{g, b} \longrightarrow \mathcal{N}_{g+1, b} \\
\beta: \mathcal{N}_{g, b} \longrightarrow \mathcal{N}_{g, b+1}
\end{aligned}
$$

and $\beta$ has a left inverse obtained by gluing a disc over a boundary component

$$
\delta: \mathcal{N}_{g, b+1} \longrightarrow \mathcal{N}_{g, b}
$$

We quote below the theorem of Wahl on the effect on these maps on group homology.

Theorem 2.1 (Theorem A of [16]) For $b \geq 1$, on homology:

(1) $\alpha$ gives a surjection in degrees $* \leq g / 4$ and an isomorphism in degrees $* \leq$ $(g-3) / 4$. 
(2) $\beta$ gives an isomorphism in degrees $* \leq(g-3) / 4$.

(3) $\delta$ gives a surjection in degrees $* \leq(g-1) / 4$ and an isomorphism in degrees $* \leq(g-5) / 4$.

We will say "the stable range" to indicate the range $* \leq(g-3) / 4$ in which $\alpha$ induces an isomorphism.

\subsection{The homotopy type of the stable nonorientable mapping class group}

Once we have homological stability for $\mathcal{N}_{g}$, the machinery of Galatius, Madsen, Tillmann and Weiss [8] identifies the stable homology of these groups.

In order to describe the result we must first introduce the spectrum $\operatorname{MTO}(2)$. Let $U_{n}^{\perp} \longrightarrow \mathrm{Gr}_{2}\left(\mathbb{R}^{n+2}\right)$ be the $n$-dimensional complement to the tautological 2-plane bundle $U_{2}$. The inclusion $\mathrm{Gr}_{2}\left(\mathbb{R}^{n+2}\right) \longrightarrow \mathrm{Gr}_{2}\left(\mathbb{R}^{n+1+2}\right)$ pulls back $U_{n+1}^{\perp}$ to $\epsilon^{1} \oplus U_{n}^{\perp}$, so there is an induced map on Thom spaces

$$
\operatorname{Th}\left(\epsilon^{1} \oplus U_{n}^{\perp}\right) \cong S^{1} \wedge \operatorname{Th}\left(U_{n}^{\perp}\right) \longrightarrow \operatorname{Th}\left(U_{n+1}^{\perp}\right) .
$$

This defines the spectrum MTO(2) with $(n+2)$-nd space $\operatorname{Th}\left(U_{n}^{\perp}\right)$. It is not hard to check that $\pi_{0}(\mathbf{M T O}(2)) \cong \mathbb{Z}$, using the cofibre sequences of spectra ((2-2) and (2-1)) in the next section.

Let $N_{g} \longrightarrow E \longrightarrow B$ be a smooth fibre bundle with fibre $N_{g}$ a nonorientable surface of genus $g$. There is an embedding of $E$ in $\mathbb{R}^{n+2} \times B$ over $B$, with a tubular neighbourhood homeomorphic to $N_{v} E$, the vertical normal bundle of $E$. A PontrjaginThom collapse gives a map

$$
S^{n+2} \wedge B_{+} \longrightarrow \operatorname{Th}\left(N_{v} E\right)
$$

and the vertical normal bundle can be classified by a map $f: E \longrightarrow G_{2}\left(\mathbb{R}^{n+2}\right)$ such that $f^{*} U_{n}^{\perp} \cong N_{v} E$. Thus $f^{*} U_{2}$ is $T_{v} E$, the vertical tangent bundle. Composing with the classifying map we have a map

$$
S^{n+2} \wedge B_{+} \longrightarrow \operatorname{Th}\left(U_{n}^{\perp}\right)
$$

with adjoint $B \longrightarrow \Omega^{n+2} \mathrm{Th}\left(U_{n}^{\perp}\right)$. Following this with the stabilisation map to $\Omega^{\infty} \mathbf{M T O}(2)$ defines the Madsen-Tillmann map

$$
\alpha_{E}: B \longrightarrow \Omega^{\infty} \mathbf{M T O}(2) .
$$

Applying this to the universal $F_{g}$ bundle over $B \mathcal{N}_{g}$ gives

$$
\alpha_{g}: B \mathcal{N}_{g} \longrightarrow \Omega^{\infty} \mathbf{M T O}(2)
$$


and similarly $\alpha_{\infty}$. The source spaces are connected and so land in a single component: it is not hard to see that it is the component of the Euler characteristic $\chi\left(N_{g}\right)$. However all components of an infinite loop space are homotopy equivalent, so we can translate so that the maps $\alpha_{E}$ always land in the 0 component of $\Omega^{\infty} \mathbf{M T O}(2)$. Together, Theorem B of [16] and Section 7 of [8] assert that the map

$$
\alpha_{\infty}: B \mathcal{N}_{\infty} \longrightarrow \Omega_{0}^{\infty} \mathbf{M T O}(2)
$$

is a homology equivalence.

One can immediately upgrade this theorem: Korkmaz [9] proves that for $g \geq 7$ the index 2 normal subgroup of $\mathcal{N}_{g}$ generated by Dehn twists is perfect, and that the first group homology in this range is $\mathbb{Z} / 2$, so this subgroup is the maximal perfect subgroup. There is then a map

$$
\alpha_{\infty}^{+}: B \mathcal{N}_{\infty}^{+} \longrightarrow \Omega_{0}^{\infty} \mathbf{M T O}(2)
$$

from the plus-construction with respect to this maximal perfect subgroup (obtained by extending $\alpha_{\infty}$ to the plus-construction, as the map has target an infinite loop space). This is still a homology isomorphism, and $\pi_{1}\left(\Omega_{0}^{\infty} \mathbf{M T O}(2)\right) \cong H_{1}\left(\Omega_{0}^{\infty} \mathbf{M T O}(2)\right) \cong \mathbb{Z} / 2$ so it is also a $\pi_{1}$-isomorphism, so a homotopy equivalence.

The rational cohomology of $\Omega_{0}^{\infty} \mathbf{M T O}(2)$ is not hard to determine: it is a polynomial algebra on the reductions of certain integral classes $\zeta_{i} \in H^{4 i}\left(\Omega_{0}^{\infty} \mathbf{M T O}(2) ; \mathbb{Z}\right)$. The author and $\mathbf{J}$ Ebert [6] have studied the divisibility of these classes in the integral cohomology of the stable nonorientable mapping class group: they are indivisible.

\subsection{The spectra MTX $(d)$ and tools from stable homotopy theory}

There are two less well-known spectra appearing in this paper, all of the form $\mathbf{M T O}(d)$. The first is MTO(2) and has been introduced in the last section. The second is MTO $(1)^{1}$ which is constructed in the same way using the unoriented Grassmannians $\mathrm{Gr}_{1}\left(\mathbb{R}^{n+1}\right)$. A full description of these spectra is available in [8]. We collect here some of their properties.

For a spectrum $\mathbf{E}=\left\{E_{n}\right\}$ the associated infinite loop space is

$$
\Omega^{\infty} \mathbf{E}:=\text { hocolim } \Omega^{n} E_{n}
$$

where the homotopy colimit, or mapping telescope, is taken using the adjoints $E_{n} \longrightarrow$ $\Omega E_{n+1}$ of the structure maps. This construction sends cofibre sequences of spectra to

\footnotetext{
${ }^{1}$ This spectrum is also known in homotopy theory as $\mathbb{R P}_{-1}^{\infty}$, though we do not use this notation.
} 
fibre sequences of infinite loop spaces. By [8] there are cofibre sequences of spectra

$$
\begin{aligned}
& \text { MTO(2) } \stackrel{\omega}{\longrightarrow} \Sigma^{\infty} \mathrm{BO}_{2+} \stackrel{\partial}{\longrightarrow} \operatorname{MTO}(1) \\
& \operatorname{MTO}(1) \stackrel{\bar{\omega}}{\longrightarrow} \Sigma^{\infty} B O_{1+} \stackrel{\bar{\partial}}{\longrightarrow} \Sigma^{\infty} S^{0}
\end{aligned}
$$

and so fibre sequences of associated infinite loop spaces. It should be noted that $\pi_{0}(\mathbf{M T O}(1))=0$, but we will still write $\Omega_{0}^{\infty} \mathbf{M T O}(1)$ to remind the reader that this space is connected.

The evaluation maps $\Sigma^{n} \Omega^{n} E_{n} \longrightarrow E_{n}$ give maps on reduced homology

$$
\tilde{H}_{*}\left(\Omega^{n} E_{n}\right) \cong \tilde{H}_{*+n}\left(\Sigma^{n} \Omega^{n} E_{n}\right) \longrightarrow \tilde{H}_{*+n}\left(E_{n}\right)
$$

and taking direct limits on both sides gives a map

$$
\sigma_{*}: \tilde{H}_{*}\left(\Omega^{\infty} \mathbf{E}\right) \longrightarrow H_{*}^{\mathrm{spec}}(\mathbf{E})
$$

to the spectrum homology of $\mathbf{E}$. This is known as the homology suspension. There is an analogous cohomology suspension

$$
\sigma^{*}: H_{\text {spec }}^{*}(\mathbf{E}) \longrightarrow \tilde{H}^{*}\left(\Omega^{\infty} \mathbf{E}\right) .
$$

If $\mathbf{E}$ is the suspension spectrum of a based space $X$, then $H_{*}^{\mathrm{spec}}(\mathbf{E}) \cong \widetilde{H}_{*}(X)$ and the inclusion

$$
i_{*}: \tilde{H}_{*}(X) \longrightarrow \tilde{H}_{*}(Q(X))=\tilde{H}_{*}\left(\Omega^{\infty} \mathbf{E}\right)
$$

gives a right inverse for $\sigma_{*}$. Similarly for the cohomology suspension.

For a proper smooth fibre bundle $p: E \longrightarrow X$ with fibre $F$ (in other words, $F$ is a compact smooth manifold without boundary), there is a stable map $\operatorname{trf}_{p}: \Sigma^{\infty} X_{+} \longrightarrow$ $\Sigma^{\infty} E_{+}$the Becker-Gottlieb transfer. There is a simple description in the case that the base $X$ is compact: then there is an embedding of $E$ into $X \times \mathbb{R}^{n}$ over $X$, for some $n$. Choose a tubular neighbourhood $U$ of $E$ in $X \times \mathbb{R}^{n}$, which is diffeomorphic to the normal bundle of $E$ in $X \times \mathbb{R}^{n}$. Restricted to a fibre $F_{x}$ embedded in $\mathbb{R}^{n}$ this bundle is simply the normal bundle, so the direct sum with the vertical tangent bundle $T_{v} E$ is trivial. Thus we write $-T_{v} E$ for the bundle diffeomorphic to $U$. A Pontrjagin-Thom collapse map gives the pretransfer

$$
\text { prt: } \Sigma^{n}\left(X_{+}\right) \longrightarrow U^{+} \cong \operatorname{Th}\left(-T_{v} E\right) .
$$

The inclusion map $-T_{v} E \longrightarrow T_{v} E \oplus-T_{v} E$ is proper, so on one-point compactification gives the inclusion

$$
\text { inc: } \operatorname{Th}\left(-T_{v} E\right) \longrightarrow \Sigma^{n}\left(E_{+}\right) \text {. }
$$


The Becker-Gottlieb transfer is the composition inc o prt: $\Sigma^{n}\left(X_{+}\right) \longrightarrow \Sigma^{n}\left(E_{+}\right)$, or its extension to suspension spectra. If the base $X$ is not compact but admits an exhaustion by compact subspaces, then one has a similar definition. In this case one only obtains a map on suspension spectra.

We gather below some results on the Becker-Gottlieb transfer.

Lemma 2.2 [1; 3, Equation (2.3), page 137] Let $p$ be a fibre bundle as above. The composition of the induced maps in cohomology $\operatorname{trf}_{p}^{*} \circ p^{*}$ is multiplication by $\chi(F)$, the Euler characteristic of the fibre. Furthermore, if $q: \widetilde{E} \longrightarrow E$ is another smooth proper fibre bundle, then $p \circ q: \widetilde{E} \longrightarrow X$ is also a smooth proper fibre bundle and $\operatorname{trf}_{p \circ q}$ and $\operatorname{trf}_{p} \circ \operatorname{trf}_{q}$ are homotopic.

If $E$ is oriented then the effect of the transfer on cohomology is given by

$$
\operatorname{trf}_{p}^{*}(x)=p_{!}\left(x \cdot e\left(T_{v} E\right)\right)
$$

where $p_{!}$is the Gysin or fibre-integration map corresponding to $p$, and $e\left(T_{v} E\right)$ is the Euler class of the vertical tangent bundle.

\subsection{Universal definition of characteristic classes}

If $c \in H^{i}\left(\mathrm{BO}_{2} ; R\right)$ is a characteristic class of unoriented 2-plane bundles we can define a characteristic class of unoriented surface bundles: let $E \stackrel{\pi}{\longrightarrow} B$ be a surface bundle then $v_{c}(E):=\operatorname{trf}_{\pi}^{*}\left(c\left(T_{v} E\right)\right) \in H^{i}(B ; R)$ is a characteristic class, by the naturality properties of the transfer. Classes that arise in this manner have a good interpretation in terms of the spectrum MTO(2).

Definition 2.3 Define a cohomology class $\bar{v}_{c}$ in $\Omega_{0}^{\infty} \mathbf{M T O}(2)$ by the image of $c$ under

$$
H^{*}\left(B O_{2} ; R\right) \stackrel{\sigma^{*}}{\longrightarrow} H^{*}\left(Q_{0}\left(B O_{2+}\right) ; R\right) \stackrel{\omega^{*}}{\longrightarrow} H^{*}\left(\Omega_{0}^{\infty} \operatorname{MTO}(2) ; R\right) .
$$

Theorem 2.4 If $\alpha_{E}: B \longrightarrow \Omega_{0}^{\infty} \mathbf{M T O}(2)$ is the Madsen-Tillmann map for a surface bundle $E \stackrel{\pi}{\longrightarrow} B$ then $\alpha_{E}^{*}\left(\bar{v}_{c}\right)=v_{c}(E)$. Thus the $\bar{v}_{c}$ are universal characteristic classes, in the sense that they do not depend on the genus of the fibre of $\pi$. 
Proof Let $T: E \rightarrow B O_{2}$ classify $T_{v} E$, and $\eta: \operatorname{Th}\left(-T_{v} E\right) \longrightarrow \mathbf{M T O}(2)$ be given by the maps classifying each vertical normal bundle. Then the diagram below commutes.

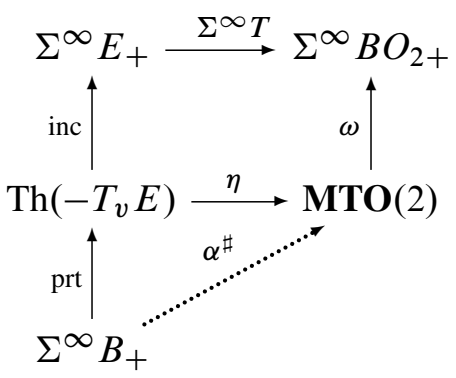

By definition $v_{c}=\operatorname{trf}^{*}\left(c\left(T_{v} E\right)\right)$, and so it is also given by $\left(\alpha^{\sharp}\right)^{*} \omega^{*}(c)$. We wish to identify $v_{c}$ via maps of spaces. Note that the adjoint of $\alpha^{\#}$ is the map $\alpha_{E}$. The following diagram commutes by the naturality of the cohomology suspension.

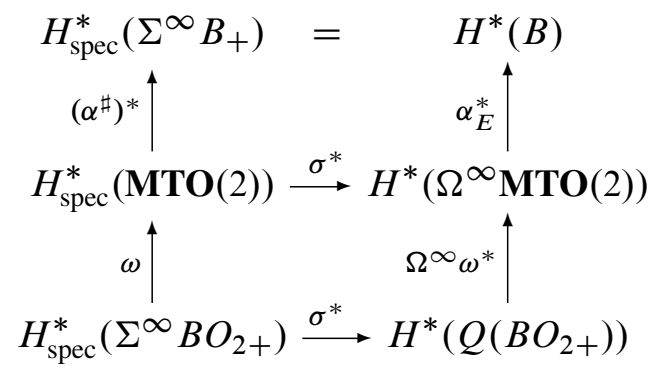

Thus $v_{c}=\alpha_{E}^{*}\left(\Omega^{\infty} \omega\right)^{*} \sigma^{*}(c)=\alpha_{E}^{*}\left(\bar{v}_{c}\right)$.

Example 2.5 The powers of the first Pontrjagin class $p_{1}^{i} \in H^{4 i}\left(\mathrm{BO}_{2} ; \mathbb{Z}\right)$ give the characteristic classes $\zeta_{i}$ defined by Wahl [16].

Example 2.6 The Stiefel-Whitney classes $w_{1}^{i} w_{2}^{j} \in H^{i+2 j}\left(\mathrm{BO}_{2} ; \mathrm{F}_{2}\right)$ define characteristic classes $\mu_{i, j}$. Even the classes $\mu_{i, 0}$ are difficult to analyse. They satisfy relations $\mu_{i, 0}^{2}=\mu_{2 i, 0}$, as the cohomology suspension $\sigma^{*}$ commutes with Steenrod squares, but the algebraic dependence between the odd classes $\mu_{2 i+1,0}$ is not known.

Example 2.7 The same result is true in the oriented case, with $\mathrm{BO}_{2}$ replaced by $\mathrm{BSO}_{2}$. Here the powers of the first Chern class $c_{1}^{i} \in H^{2 i}\left(\mathrm{BSO}_{2} ; \mathbb{Z}\right)$ define integral characteristic classes $\kappa_{i}$ which in the oriented mapping class groups give the MillerMorita-Mumford classes.

\subsection{Hopf algebras}

We will make much use of the structure of Hopf algebras over a field $\mathbb{F}$. Let us fix notation, which coincides with that of the classic reference on Hopf algebras of Milnor 
and Moore [14]. Recall that an abelian Hopf algebra is one that is commutative and cocommutative. All Hopf algebras appearing in this paper are homology groups of infinite loop spaces and as such are abelian. We write $\psi: X \longrightarrow X \otimes X$ for the coproduct of a coalgebra $X$, and $\psi_{Y}: Y \longrightarrow X \otimes Y$ for the coaction of the coalgebra $X$ on a right comodule $Y$.

For a coalgebra $A$, a left $A$-comodule $B$ and a right $A$-comodule $C$, the cotensor product $B \square_{A} C$ is defined as the kernel of

$$
B \otimes C \stackrel{\psi_{B} \otimes \mathrm{id}_{C}-\mathrm{id}_{B} \otimes \psi_{C}}{\longrightarrow} B \otimes A \otimes C .
$$

In general $B \square_{A} C$ is just a vector space, but if $A$ is cocommutative then it is again an $A$-comodule.

The Hopf algebra kernel and cokernel of a map $f: A \longrightarrow B$ of Hopf algebras are $A \rrbracket f:=A \square_{B} \mathbb{F}$ and $B / / f:=B \otimes_{A} \mathbb{F}$ respectively. When $A$ and $B$ are abelian, kernels and cokernels are again (abelian) Hopf algebras. We will write $P A$ and $Q A$ for the vector spaces of primitive and indecomposable elements respectively.

We will make use of two corollaries of Borel's structure theorem for finite-type abelian Hopf algebras over a perfect field [14, Theorem 7.11].

Lemma 2.8 Firstly, a Hopf subalgebra of a polynomial algebra is again polynomial. Secondly, if $f: A \longrightarrow B$ is an injective homomorphism of Hopf algebras, then $B$ is a free $A$-module. Dually, if $f$ is surjective then $A$ is a free $B$-comodule.

\subsection{The Eilenberg-Moore spectral sequence}

In this section all homology is with coefficients in some field $\mathbb{F}$. For a fibration sequence $F \longrightarrow E \stackrel{\pi}{\longrightarrow} B$ of connected spaces, the Eilenberg-Moore spectral sequence is

$$
E_{p, q}^{2}=\operatorname{Cotor}_{p, q}^{H_{*}(B)}\left(H_{*}(E), \mathbb{F}\right) \Rightarrow H_{p+q}(F)
$$

where the subscript on Cotor denotes the degree $q$ part of the $p$-th derived functor of $-\square_{H_{*}(B)} \mathbb{F}$. The $H_{*}(B)$-comodule structure on $H_{*}(E)$ is induced by the map $\pi_{*}$. The usual requirement for convergence is that $B$ be simply connected, but we will use the exotic convergence of [5]: base spaces will have $\mathbb{Z} / 2$ as fundamental group, which always acts nilpotently on any finitely-generated $\mathbb{F}_{2}$-homology group.

When the fibration is one of connected components of infinite loop spaces,

$$
\Omega_{0}^{\infty} \mathbf{F} \longrightarrow \Omega_{0}^{\infty} \mathbf{E} \stackrel{\pi}{\longrightarrow} \Omega_{0}^{\infty} \mathbf{B}
$$


coming from a cofibre sequence of finite-type spectra, all the $\mathbb{F}$-homology groups are finite-type abelian Hopf algebras over $\mathbb{F}$. Suppose that some condition guaranteeing the convergence of the Eilenberg-Moore spectral sequence holds. If $\pi_{*}: H_{*}\left(\Omega_{0}^{\infty} \mathbf{E} ; \mathbb{F}\right) \longrightarrow$ $H_{*}\left(\Omega_{0}^{\infty} \mathbf{B} ; \mathbb{F}\right)$ is surjective then Lemma 2.8 implies that $H_{*}\left(\Omega_{0}^{\infty} \mathbf{E} ; \mathbb{F}\right)$ is a free $H_{*}\left(\Omega_{0}^{\infty} \mathbf{B} ; \mathbb{F}\right)$-comodule. Thus the $E^{2}$-term of the Eilenberg-Moore spectral sequence is

$$
\begin{aligned}
\operatorname{Cotor}_{p, q}^{H_{*}\left(\Omega_{0}^{\infty} \mathbf{B} ; \mathbb{F}\right)}\left(H_{*}\left(\Omega_{0}^{\infty} \mathbf{E} ; \mathbb{F}\right), \mathbb{F}\right) & =H_{*}\left(\Omega_{0}^{\infty} \mathbf{E} ; \mathbb{F}\right) \square_{H_{*}\left(\Omega_{0}^{\infty} \mathbf{B} ; \mathbb{F}\right) \mathbb{F}} \\
& =H_{*}\left(\Omega_{0}^{\infty} \mathbf{E} ; \mathbb{F}\right) \backslash \pi_{*}
\end{aligned}
$$

concentrated in the line $p=0$, so the spectral sequence collapses. Thus

$$
\mathbb{F} \longrightarrow H_{*}\left(\Omega_{0}^{\infty} \mathbf{F} ; \mathbb{F}\right) \longrightarrow H_{*}\left(\Omega_{0}^{\infty} \mathbf{E} ; \mathbb{F}\right) \stackrel{\pi_{*}}{\longrightarrow} H_{*}\left(\Omega_{0}^{\infty} \mathbf{B} ; \mathbb{F}\right) \longrightarrow \mathbb{F}
$$

is a short exact sequence of Hopf algebras.

\subsection{Araki-Kudo operations and the homology of $Q\left(Y_{+}\right)$}

An infinite loop space $X$ has a rich structure on its $\mathbb{F}_{2}$-homology. Firstly the H-space structure gives the homology the structure of a Hopf algebra, with the Pontrjagin product denoted $*$. Secondly there are Araki-Kudo operations [10]: these are a family of homomorphisms

$$
Q^{s}: H_{i}\left(X ; \mathbb{F}_{2}\right) \longrightarrow H_{i+s}\left(X ; \mathbb{F}_{2}\right)
$$

that are natural for maps of infinite loop spaces. These have formal properties similar to the Steenrod squares: a Cartan formula and Adém relations. The reference is Cohen, Lada and May [4], and we will use many properties of these operations. We recall some elementary notions. The sequence $I=\left(s_{1}, \ldots, s_{k}\right)$ is called admissible if $s_{i} \leq 2 s_{i+1}$ for all $i$. The excess of such a sequence is the integer $e(I):=s_{1}-\sum_{i=2}^{k} s_{i}$. The length of $I$ is $l(I):=k$. The degree of $I$ is $d(I):=\sum_{i} s_{i}$. We write $Q^{I}=Q^{s_{1}} \cdots Q^{s_{k}}$. We write $\mathcal{R}$ for the algebra of all operations, and call it the Dyer-Lashof algebra.

For a space $Y$, the $\mathbb{F}_{2}$-homology of $Q\left(Y_{+}\right)$has a (non functorial) description as follows. Let $\mathcal{B}$ be a homogenous basis for $H_{*}\left(Y ; \mathbb{F}_{2}\right)$. Then the $\mathbb{F}_{2}$-homology of $Q\left(Y_{+}\right)$is the polynomial algebra on the set

$$
\left\{Q^{I}(x) \mid x \in \mathcal{B}, I \text { admissible, } e(I)>|x|\right\} .
$$

If the space $Y$ is connected, $\pi_{0}\left(Q\left(Y_{+}\right)\right) \cong \mathbb{Z}$ and we write $[n]$ for the image of $n \in \pi_{0}$ in $H_{0}\left(Q\left(Y_{+}\right)\right)$. The $\mathbb{F}_{2}$-homology of the 0 component $Q_{0}\left(Y_{+}\right)$is then the polynomial algebra on the set

$$
\left\{Q^{I}(x) *\left[-2^{l(I)}\right] \mid x \in \mathcal{B}, I \text { admissible, } e(I)>|x|,\left|Q^{I}(x)\right|>0\right\} .
$$




\section{Mod 2 homology of $\Omega_{0}^{\infty} \operatorname{MTO}(1)$}

It is clear that the map

$$
\bar{\partial}: Q_{0}\left(B O_{1+}\right) \longrightarrow Q_{0}\left(S^{0}\right)
$$

is the transfer for the double covering $E O_{1} \longrightarrow B O_{1}, t: Q\left(B O_{1+}\right) \longrightarrow Q\left(S^{0}\right)$. The effect of this map on homology has been calculated by Mann, Miller and Miller [13]:

Lemma 3.1 Let $e_{i} \in H_{i}\left(B O_{1} ; \mathbb{F}_{2}\right)$ be the unique nontrivial class. Then $t_{*}\left(e_{i}\right)=$ $Q^{i}([1])$.

Corollary 3.2 Thus the map $\bar{\partial}: Q_{0}\left(B O_{1+}\right) \longrightarrow Q_{0}\left(S^{0}\right)$ on homology is

$$
\bar{\partial}_{*}\left(e_{i} *[-1]\right)=Q^{i}([1]) *[-2]
$$

so is surjective, as these elements generate $H_{*}\left(Q_{0}\left(S^{0}\right)\right)$ over the Dyer-Lashof algebra.

Proof of Theorem A The Eilenberg-Moore spectral sequence for the fibration

$$
\Omega_{0}^{\infty} \mathbf{M T O}(1) \longrightarrow Q_{0}\left(\mathrm{BO}_{1+}\right) \stackrel{\bar{\partial}}{\longrightarrow} Q_{0}\left(S^{0}\right)
$$

converges to $H_{*}\left(\Omega_{0}^{\infty} \mathbf{M T O}(1)\right)$ as the base space has fundamental group $\mathbb{Z} / 2$. By Corollary $3.2, \bar{\partial}_{*}$ is surjective, so by the discussion in Section 2.6 we obtain the short exact sequence of Hopf algebras.

Finally, $H_{*}\left(Q_{0}\left(S^{0}\right)\right)$ is a free algebra so the sequence splits as algebras.

Remark 3.3 If $I$ is some sequence, $Q^{I}$ is an Araki-Kudo operation and one can iteratively apply Adém relations to write it as a linear combination of $Q^{J}$ with $J$ admissible. We write $Q^{I}=\sum_{J} \lambda_{J}^{I} Q^{J}$ where the $\lambda$ are the coefficients needed to express $Q^{I}$ as admissible monomials. We adopt the convention that we sum over all $J$, and that $\lambda_{J}^{I}=0$ if $J$ is not admissible. Adém relations decrease excess, so $\lambda_{J}^{I}=0$ if $e(J)>e(I)$ also.

For a sequence $I$ and an integer $i$ such that $I$ is admissible, $e(I)>i$ and $(I, i)$ is not admissible, there is an element $v^{I, i}$ in $Q H_{*}\left(Q_{0}\left(B O_{1+}\right)\right)$ given by

$$
v^{I, i}:=Q^{I}\left(e_{i}\right) *\left[-2^{1+l(I)}\right]+\sum_{J, j} \lambda_{J, j}^{I, i} Q^{J}\left(e_{j}\right) *\left[-2^{1+l(J)}\right]
$$

where the sum is over all sequences $(J, j)$. This clearly lies in the kernel of $Q\left(\bar{\partial}_{*}\right)$. We adopt the convention that $v^{I, i}$ is also defined for $(I, i)$ admissible, and is 0 . This is the natural extension, as $Q^{I} Q^{i}$ is already written in terms of admissibles. 
Theorem 3.4 The Hopf algebra $H_{*}\left(\Omega_{0}^{\infty} \mathbf{M T O}(1) ; \mathbb{F}_{2}\right)$ is polynomial on a set

$$
\left\{V^{I, i} \mid I \text { admissible, } e(I)>i,(I, i) \text { not admissible }\right\}
$$

where $\bar{\omega}_{*}\left(V^{I, i}\right)=v^{I, i}$ modulo decomposables.

Proof It is now clear that $H_{*}\left(\Omega_{0}^{\infty} \mathbf{M T O}(1)\right)$ is a polynomial algebra, as for Hopf algebras over $\mathbb{F}_{2}$ a Hopf subalgebra of a polynomial algebra is again polynomial. Furthermore as the sequence splits as algebras there is an exact sequence of indecomposable quotients

$$
0 \longrightarrow Q H_{*}\left(\Omega_{0}^{\infty} \mathbf{M T O}(1)\right) \stackrel{Q\left(\bar{\omega}_{*}\right)}{\longrightarrow} Q H_{*}\left(Q_{0}\left(B O_{1+}\right)\right) \stackrel{Q\left(\bar{\partial}_{*}\right)}{\longrightarrow} Q H_{*}\left(Q_{0}\left(S^{0}\right)\right) \longrightarrow 0 .
$$

The elements $v^{I, i}$ described earlier are indecomposable and lie in the kernel of $Q\left(\bar{\partial}_{*}\right)$. Thus there are polynomial generators $V^{I, i}$ such that $Q\left(\bar{\omega}_{*}\right)\left(V^{I, i}\right)=v^{I, i}$. The theorem follows from the following Lemma.

Lemma 3.5 The $v^{I, i}$ form an additive basis of $\operatorname{Ker}\left(Q\left(\bar{\partial}_{*}\right)\right)$.

Proof Let $v=\sum \mu_{I, i} Q^{I}\left(e_{i}\right) *\left[-2^{1+l(I)}\right]$ be an element in the kernel of $Q\left(\bar{\partial}_{*}\right)$. Then

$$
\begin{aligned}
0=\bar{\partial}_{*}(v) & =\sum_{I, i} \mu_{I, i} Q^{I} Q^{i}([1]) *\left[-2^{1+l(I)}\right] \\
& =\sum_{I, i} \mu_{I, i} \sum_{J, j} \lambda_{J, j}^{I, i} Q^{J} Q^{j}([1]) *\left[-2^{1+l(J)}\right] \\
& =\sum_{J, j}\left(\sum_{I, i} \mu_{I, i} \lambda_{J, j}^{I, i}\right) Q^{J} Q^{j}([1]) *\left[-2^{1+l(J)}\right] .
\end{aligned}
$$

so $\sum_{I, i} \mu_{I, i} \lambda_{J, j}^{I, i}=0$ for each $(J, j)$ admissible and of positive excess. Now consider

$$
\begin{aligned}
v-\sum_{I, i} \mu_{I, i} v^{I, i} & =\sum_{I, i} \mu_{I, i}\left(\sum_{J, j} \lambda_{J, j}^{I, i} Q^{J}\left(e_{j}\right)\right) *\left[-2^{1+l(J)}\right] \\
& =\sum_{J, j}\left(\sum_{I, i} \mu_{I, i} \lambda_{J, j}^{I, i}\right) Q^{J}\left(e_{j}\right) *\left[-2^{1+l(J)}\right] \\
& =0 .
\end{aligned}
$$

So the $v^{I, i}$ span the kernel of the map $Q\left(\bar{\partial}_{*}\right)$. 
Now let $\sum_{I, i} \mu_{I, i} v^{I, i}=0$, so

$$
\begin{aligned}
\sum \mu_{I, i} Q^{I}\left(e_{i}\right) *\left[-2^{1+l(I)}\right] & =\sum_{I, i} \mu_{I, i} \sum_{J, j} \lambda_{J, j}^{I, i} Q^{J}\left(e_{j}\right) *\left[-2^{1+l(J)}\right] \\
& =\sum_{J, j}\left(\sum_{I, i} \mu_{I, i} \lambda_{J, j}^{I, i}\right) Q^{J}\left(e_{j}\right) *\left[-2^{1+l(J)}\right] .
\end{aligned}
$$

If $(I, i)$ is not admissible then $Q^{I}\left(e_{i}\right) *\left[-2^{1+l(I)}\right]$ does not appear on the right hand side, so $\mu_{I, i}=0$. So all the coefficients are 0 and the $v^{I, i}$ are linearly independent. $\square$

It will also be important later to understand the action of the Araki-Kudo operations on the polynomial generators $v^{I, i}$ and $V^{I, i}$.

Theorem 3.6 If $(l, I)$ is an admissible sequence then in $Q H_{*}\left(Q_{0}\left(B O_{2+}\right)\right)$

$$
Q^{l}\left(v^{I, i}\right)=v^{(l, I), i}+\sum_{j, J, J^{\prime}} \lambda_{J, j}^{I, i} \lambda_{J^{\prime}}^{l, J} v^{J^{\prime}, j}
$$

and the same formula holds for $V^{I, i}$ in $Q H_{*}\left(\Omega_{0}^{\infty} \mathbf{M T O}(1)\right)$. Furthermore, if $\lambda_{J, j}^{I, i} \neq 0$ then $j>i$.

Proof Firstly we analyse the effect of $Q^{l}$ on $v^{I, i} *\left[2^{1+l(I)}\right]$.

$$
\begin{aligned}
Q^{l}\left(v^{I, i} *\left[2^{1+l(I)}\right]\right) & =Q^{l} Q^{I}\left(e_{i}\right)+\sum_{J, j} \lambda_{J, j}^{I, i} Q^{l} Q^{J}\left(e_{j}\right) \\
& =Q^{(l, I)}\left(e_{i}\right)+\sum_{J, j} \lambda_{J, j}^{I, i} \sum_{J^{\prime}} \lambda_{J^{\prime}}^{l, J} Q^{J^{\prime}}\left(e_{j}\right) .
\end{aligned}
$$

Note that this element is in the kernel of $\bar{\partial}_{*}$, as $v^{I, i}$ is and $\bar{\partial}_{*}$ commutes with ArakiKudo operations and the Pontrjagin product. Thus

$$
\begin{aligned}
0 & =Q^{(l, I)} Q^{i}([1])+\sum_{J^{\prime}, J, j} \lambda_{J, j}^{I, i} \lambda_{J^{\prime}}^{l, J} Q^{J^{\prime}} Q^{j}([1]) \\
& =Q^{(l, I, i)}([1])+\sum_{J^{\prime \prime}, J^{\prime}, J, j} \lambda_{J, j}^{I, i} \lambda_{J^{\prime}}^{l, J} \lambda_{J^{\prime \prime}}^{J^{\prime}, j} Q^{J^{\prime \prime}}([1])
\end{aligned}
$$

and the terms in the summation are all admissible. Thus this is the unique way to express $Q^{(l, I, i)}$ in terms of admissible operations, so

$$
\lambda_{J^{\prime \prime}}^{l, I, i}=\sum_{J^{\prime}, J, j} \lambda_{J, j}^{I, i} \lambda_{J^{\prime}}^{l, J} \lambda_{J^{\prime \prime}}^{J^{\prime}, j} .
$$


Secondly we analyse the expression for $v^{(l, I), i} *\left[2^{2+l(I)}\right]$.

$$
\begin{aligned}
v^{(l, I), i} *\left[2^{2+l(I)}\right] & =Q^{(l, I)}\left(e_{i}\right)+\sum_{J^{\prime \prime \prime}, j^{\prime}} \lambda_{J^{\prime \prime \prime}, j^{\prime}}^{l, I, i} Q^{J^{\prime \prime \prime}}\left(e_{j^{\prime}}\right) \\
& =Q^{(l, I)}\left(e_{i}\right)+\sum_{J^{\prime \prime \prime}, j^{\prime}}\left(\sum_{J^{\prime}, J, j} \lambda_{J, j}^{I, i} \lambda_{J^{\prime}}^{l, J} \lambda_{J^{\prime \prime \prime}, j^{\prime}}^{J^{\prime}, j}\right) Q^{J^{\prime \prime \prime}}\left(e_{j^{\prime}}\right) \\
& =Q^{(l, I)}\left(e_{i}\right)+\sum_{J^{\prime}, J, j} \lambda_{J_{, j}, i, i}^{I, J} \lambda_{J^{\prime}}^{l, J}\left(\sum_{J^{\prime \prime \prime}, j^{\prime}} \lambda_{J^{\prime \prime \prime}, j^{\prime}}^{J^{\prime}, j} Q^{J^{\prime \prime \prime}}\left(e_{j^{\prime}}\right)\right) \\
& =Q^{(l, I)}\left(e_{i}\right)+\sum_{J^{\prime}, J, j} \lambda_{J_{, j}, i}^{I, i} \lambda_{J^{\prime}}^{l, J}\left(v^{J^{\prime}, j} *\left[2^{1+l\left(J^{\prime}\right)}\right]+Q^{J^{\prime}}\left(e_{j}\right)\right) \\
& =Q^{(l, I)}\left(e_{i}\right)+\sum_{J, j} \lambda_{J, j}^{I, i} Q^{(l, J)}\left(e_{j}\right) \\
& +\sum_{J^{\prime}, J, j} \lambda_{J, j}^{I, i} \lambda_{J^{\prime}}^{l, J} v^{J^{\prime}, j} *\left[2^{1+l\left(J^{\prime}\right)}\right] \\
& =Q^{l}\left(v^{I, i} *\left[2^{1+l(I)}\right]\right)+\sum_{J^{\prime}, J, j} \lambda_{J_{, j}, i}^{I, i} \lambda_{J^{\prime}}^{l, J} v^{J^{\prime}, j} *\left[2^{1+l\left(J^{\prime}\right)}\right] .
\end{aligned}
$$

Then $Q^{l}\left(v^{I, i} *\left[2^{1+l(I)}\right]\right)=Q^{l}\left(v^{I, i}\right) *\left[2^{2+l(I)}\right]$ modulo decomposables, so the result follows after translating back to the 0 component, as $l\left(J^{\prime}\right)=l(I)+1$.

Finally, as $(I, i)$ is not admissible but $I$ is, to write $Q^{(I, i)}$ as admissibles an Adém relation must be applied involving the last term $Q^{i}$. Applying an Adém relation to $Q^{a} Q^{b}$ gives monomials $Q^{A} Q^{B}$ with $B$ strictly larger than $b$, so if $\lambda_{J, j}^{I, i} \neq 0$ then $j>i$.

\section{The map $\partial$ on homology}

We wish to study the composition

$$
Q\left(\mathrm{BO}_{2+}\right) \stackrel{\partial}{\longrightarrow} \Omega^{\infty} \mathbf{M T O}(1) \stackrel{\bar{\omega}}{\longrightarrow} Q\left(\mathrm{BO}_{1+}\right)
$$

and as $\partial$ is the pretransfer for the circle bundle $S^{1} \longrightarrow \mathrm{BO}_{1} \longrightarrow \mathrm{BO}_{2}$, it then follows that $\bar{\omega} \circ \partial$ is the transfer for this bundle.

Remark 4.1 In general there are fibre bundles $S^{n-1} \longrightarrow \mathbb{S}\left(\gamma_{n}\right) \stackrel{\pi}{\longrightarrow} B O_{n}$ and a map $f: \mathbb{S}\left(\gamma_{n}\right) \longrightarrow B O_{n-1}$ classifying the vertical tangent bundle. Then the composition

$$
Q\left(B O_{n+}\right) \stackrel{\operatorname{trf}_{\pi}}{\longrightarrow} Q\left(\mathbb{S}\left(\gamma_{n}\right)_{+}\right) \stackrel{f}{\longrightarrow} Q\left(B O_{n-1+}\right)
$$


coincides with the composition

$$
Q\left(B O_{n+}\right) \stackrel{\partial}{\longrightarrow} \Omega^{\infty} \mathbf{M T O}(n-1) \stackrel{\omega}{\longrightarrow} Q\left(B O_{n-1+}\right)
$$

of maps occurring in the analogs of the fibrations (2-1) and (2-2).

To evaluate this transfer we will use the technique of Brumfiel and Madsen [3] of reducing it to a transfer for a finite-sheeted cover. Thus we must find a nondegenerate vector field on the fibre that is equivariant for the action of the structure group. We can not do this directly as $S^{1}$ does not admit any $O_{2}$-invariant nondegenerate vector fields, so instead we consider the pullback bundle via $d: \mathrm{BO}_{1} \times \mathrm{BO}_{1} \longrightarrow \mathrm{BO}_{2}$, the map classifying $\gamma_{1} \times \gamma_{1}$,

$$
S^{1} \longrightarrow \mathbb{S}\left(\gamma_{1} \times \gamma_{1}\right) \longrightarrow B O_{1} \times B O_{1} .
$$

There is a nondegenerate $O_{1} \times O_{1}$-invariant vector field on $S^{1}$. It has 4 singular points split into two orbits of two each, having opposite indices. Thus the singular locus $\Sigma \subset \mathbb{S}\left(\gamma_{1} \times \gamma_{1}\right)$ is simply $E O_{1} \times B O_{1} \amalg B O_{1} \times E O_{1}$. We can thus compute the transfer for this bundle in terms of the transfer map $t$ of Section 3.

Proposition 4.2 The composition $B O_{1} \times B O_{1} \stackrel{d}{\rightarrow} B O_{2} \stackrel{T}{\rightarrow} Q\left(B O_{1+}\right)$ is homotopic to

$$
\begin{aligned}
B O_{1} \times B O_{1} \stackrel{\Delta}{\longrightarrow} B O_{1} \times B O_{1} \times B O_{1} \times B O_{1} \\
\stackrel{(t \wedge \mathrm{id}) \times(\mathrm{id} \wedge t)}{\longrightarrow} Q_{2}\left(E O_{1} \times B O_{1+}\right) \times Q_{2}\left(B O_{1} \times E O_{1+}\right) \\
\stackrel{\mathrm{id} \times \chi}{\longrightarrow} Q_{2}\left(B O_{1+}\right) \times Q_{-2}\left(B O_{1+}\right) \stackrel{\mu}{\longrightarrow} Q_{0}\left(B O_{1+}\right) .
\end{aligned}
$$

Proof This is an elementary application of Theorem 2.10 of [3]. We are aware that there has been a correction to this paper in [11], but our indices are very simple and there is no difference.

We can now effectively identify the map $\partial_{*}$. The map $d_{*}$ is surjective and we proved in the last section that $\bar{\omega}_{*}$ is injective, so the map $T_{*} \circ d_{*}$ determines $\partial_{*}$.

Theorem 4.3 The composition

$$
H_{*}\left(B O_{1} \times B O_{1}\right) \stackrel{d_{*}}{\longrightarrow} H_{*}\left(B O_{2}\right) \longrightarrow H_{*}\left(Q_{1}\left(B O_{2+}\right)\right) \stackrel{\bar{\omega}_{*} \partial_{*}}{\longrightarrow} H_{*}\left(Q_{0}\left(B O_{1+}\right)\right)
$$


is given by

$$
e_{i} \otimes e_{j} \mapsto \sum_{a=0}^{i} \sum_{b=0}^{j} \sum_{s=0}^{b} \sum_{t=0}^{a}\left(\begin{array}{c}
b-s \\
s
\end{array}\right)\left(\begin{array}{c}
a-t \\
t
\end{array}\right) Q^{i-a+s}\left(e_{b-s}\right) * \chi\left(Q^{j-b+t}\left(e_{a-t}\right)\right)
$$

which modulo decomposables is

$$
\sum_{s=0}^{j}\left(\begin{array}{c}
j-s \\
s
\end{array}\right) Q^{i+s}\left(e_{j-s}\right) *[-2]+\sum_{t=0}^{i}\left(\begin{array}{c}
i-t \\
t
\end{array}\right) Q^{j+t}\left(e_{i-t}\right) *[-2] .
$$

Proof Applying the description of the composition from the previous proposition gives

$$
e_{i} \otimes e_{j} \mapsto \sum_{a=0}^{i} \sum_{b=0}^{j}\left(Q^{i-a}([1]) \wedge e_{b}\right) * \chi\left(e_{a} \wedge Q^{j-b}([1])\right) .
$$

We then use the following formulae from [4] for evaluating the smash product on homology:

$$
\begin{aligned}
& (a * b) \wedge c=\sum\left(a \wedge c^{\prime}\right) *\left(b \wedge c^{\prime \prime}\right) \text { when } \Delta_{*}(c)=\sum c^{\prime} \otimes c^{\prime \prime} \\
& Q^{i}(x) \wedge y=\sum Q^{i+t}\left(x \wedge S q_{*}^{t}(y)\right)
\end{aligned}
$$

So

$$
Q^{i-a}([1]) \wedge e_{b}=\sum_{s=0}^{b} Q^{i-a+s}\left([1] \wedge S q_{*}^{s}\left(e_{b}\right)\right)
$$

which can be expressed as

$$
\sum_{s=0}^{b}\left(\begin{array}{c}
b-s \\
s
\end{array}\right) Q^{i-a+s}\left(e_{b-s}\right)
$$

as $S q_{*}^{s}\left(e_{b}\right)=\left(\begin{array}{c}b-s \\ s\end{array}\right) e_{b-s}$. This gives the first expression. The expression modulo decomposables follows immediately, using the fact that $\chi\left(e_{i}\right)=e_{i} *[-2]$ modulo decomposables.

Corollary 4.4 The formula in Theorem 4.3 for the indecomposable part of the image of $e_{i} \otimes e_{j}$ lies in the kernel of $Q\left(\bar{\partial}_{*}\right)$ and so can be expressed as a linear combination of the $v^{I, i}$. This expression is simply

$$
\sum_{s=0}^{j}\left(\begin{array}{c}
j-s \\
s
\end{array}\right) v^{i+s, j-s}+\sum_{t=0}^{i}\left(\begin{array}{c}
i-t \\
t
\end{array}\right) v^{j+t, i-t}
$$

Thus $e_{0} \otimes e_{i}$ maps to $v^{i, 0}$ modulo decomposables. 
Proof Similarly to the proof of Lemma 3.5, consider

$$
Q\left(\partial_{*} \bar{\omega}_{*} d_{*}\right)\left(e_{i} \otimes e_{j}\right)+\sum_{s=0}^{j}\left(\begin{array}{c}
j-s \\
s
\end{array}\right) v^{i+s, j-s}+\sum_{t=0}^{i}\left(\begin{array}{c}
i-t \\
t
\end{array}\right) v^{j+t, i-t}
$$

which by the definition of the $v^{I, i}$ can be written as

$$
\sum_{s=0}^{j}\left(\begin{array}{c}
j-s \\
s
\end{array}\right) \sum_{a, b} \lambda_{a, b}^{i+s, j-s} Q^{a}\left(e_{b}\right) *[-2]+\sum_{t=0}^{i}\left(\begin{array}{c}
i-t \\
t
\end{array}\right) \sum_{a, b} \lambda_{a, b}^{j+t, i-t} Q^{a}\left(e_{b}\right) *[-2]
$$

so the coefficient of $Q^{a}\left(e_{b}\right) *[-2]$ is

$$
\sum_{s=0}^{j}\left(\begin{array}{c}
j-s \\
s
\end{array}\right) \lambda_{a, b}^{i+s, j-s}+\sum_{t=0}^{i}\left(\begin{array}{c}
i-t \\
t
\end{array}\right) \lambda_{a, b}^{j+t, i-t}
$$

This element lies in the kernel of $\bar{\partial}_{*}$, and the $Q^{a}\left(e_{b}\right)$ are sent to $Q^{(a, b)}([1]) *[-4]$ where $(a, b)$ is admissible. These are linearly independent in the homology of $Q_{0}\left(S^{0}\right)$ and so all the coefficients are 0 . Thus the expression above is identically 0 , and the result follows.

Proposition 4.5 The map $Q\left(\partial_{*}\right): Q H_{*}\left(Q_{0}\left(B O_{2+}\right)\right) \longrightarrow Q H_{*}\left(\Omega_{0}^{\infty} \mathbf{M T O}(1)\right)$ is surjective.

Proof Define an increasing filtration $G^{i}:=\left\langle V^{a, b} \mid b \leq i\right\rangle$ of $G^{\infty}:=\left\langle V^{a, b}\right\rangle \subset$ $Q H_{*}\left(\Omega_{0}^{\infty} \mathbf{M T O}(1)\right)$. The previous corollary implies that $G^{0}$ is in the image of $Q\left(\partial_{*}\right)$. Consider the indecomposable element $V^{i-a, a}$, so $i-a>2 a$, and $a<i / 3$. Then

$$
Q\left(\partial_{*} d_{*}\right)\left(e_{a} \otimes e_{i-a}\right)=\sum_{s=0}^{i-a}\left(\begin{array}{c}
i-a-s \\
s
\end{array}\right) V^{a+s, i-a-s}+\sum_{t=0}^{a}\left(\begin{array}{c}
a-t \\
t
\end{array}\right) V^{i-a+t, a-t} .
$$

Either $2 s+a>i$, so $s>i-a-s$ and the binomial coefficient in the first sum is 0 , or $2 s+a \leq i$. This together with $a<i / 3$ implies that $a+s \leq 2(i-a-s)$, so $(a+s, i-a-s)$ is admissible and $V^{a+s, i-a-s}$ is 0 . Thus the first sum is 0 . The second sum is

$$
V^{i-a, a}+\sum_{t=1}^{a}\left(\begin{array}{c}
a-t \\
t
\end{array}\right) V^{i-a+t, a-t}
$$

so is $V^{i-a, a}$ modulo $G^{a-1}$. Thus by induction along the filtration $G^{*}, G^{\infty}=\left\langle V^{a, b}\right\rangle$ is in the image of $Q\left(\partial_{*}\right)$. The map $\partial$ is an infinite loop map, so commutes with Araki-Kudo operations: thus $\mathcal{R} \cdot G^{\infty}$ is also in the image. 
Now we introduce a new filtration $F^{i}:=\left\langle V^{I, j} \mid j \geq i\right\rangle$ of $Q H_{*}\left(\Omega_{0}^{\infty} \mathbf{M T O}(1)\right)$. This filtration is decreasing, with $F^{0}$ the whole vector space, but it has the property that in any fixed degree $n$ the filtration $F_{n}^{*}$ is has finite length. In particular

$$
F_{n}^{n}:=\left\langle V^{I, j} \mid j \geq n, d(I)+j=n\right\rangle=\{0\}
$$

as $d(I)>0$. We will proceed by induction up the filtration in each degree. By Theorem 3.6 , for $(l, I)$ admissible,

$$
Q^{l}\left(V^{I, i}\right)=V^{(l, I), i}+\sum_{j, J, J^{\prime}} \lambda_{J, j}^{I, i} \lambda_{J^{\prime}}^{l, J} V^{J^{\prime}, j}
$$

and for each term in the sum $j>i$. In particular the sum lies in $F^{i+1}$, so $Q^{l}\left(V^{I, i}\right)=$ $V^{(l, I), i}$ modulo $F^{i+1}$. Thus by iterating,

$$
V^{(I, k), i}=Q^{I}\left(V^{k, i}\right) \text { modulo } F^{i+1}
$$

and so $F^{i} \subseteq \mathcal{R} \cdot G^{\infty}+F^{i+1}$. In degree $n, F_{n}^{n}=\{0\}$ is in the image of $Q\left(\partial_{*}\right)$, so by this inclusion $F_{n}^{n-1}$ is too, and so on: thus $F_{n}^{0}$ is in the image. This holds in all degrees $n$ so $F^{0}$ is in the image, but this is the entire space $Q H_{*}\left(\Omega_{0}^{\infty} \mathbf{M T O}(1)\right)$. $\square$

\section{The homology of $\Omega_{0}^{\infty} \mathrm{MTO}(2)$}

Proof of Theorem B We now study the Eilenberg-Moore spectral sequence for the fibration

$$
\Omega_{0}^{\infty} \mathbf{M T O}(2) \longrightarrow Q_{0}\left(\mathrm{BO}_{2+}\right) \stackrel{\partial}{\longrightarrow} \Omega_{0}^{\infty} \mathbf{M T O}(1)
$$

noting that $\pi_{1}\left(\Omega_{0}^{\infty} \mathbf{M T O}(1)\right) \cong \mathbb{Z} / 2$ and so by the discussion in Section 2.6 the spectral sequence converges to $H_{*}\left(\Omega_{0}^{\infty} \mathbf{M T O}(2)\right)$. By Proposition $4.5, \partial_{*}$ is surjective, so by the discussion in Section 2.6 we obtain the short exact sequence of Hopf algebras.

Finally, $H_{*}\left(\Omega_{0}^{\infty} \mathbf{M T O}(1)\right)$ is a polynomial algebra, so free, so the sequence is split as algebras.

Corollary 5.1 It now follows that $H_{*}\left(\Omega_{0}^{\infty} \mathbf{M T O}(1) ; \mathbb{F}_{2}\right)$ is also dual to a polynomial algebra, as $H^{*}\left(\Omega_{0}^{\infty} \mathbf{M T O}(1) ; \mathbb{F}_{2}\right)$ injects into $H^{*}\left(Q_{0}\left(B O_{2+}\right) ; \mathbb{F}_{2}\right)$, which is polynomial.

\subsection{Remarks on integral (co)homology}

Write $X\left[\frac{1}{2}\right]$ for the $\mathbb{Z}\left[\frac{1}{2}\right]$-localisation in spaces or spectra. The spectrum $\operatorname{MTO}(1)\left[\frac{1}{2}\right]$ is contractible (this follows immediately from Corollary 6.3 of [16]), so

$$
\operatorname{MTO}(2)\left[\frac{1}{2}\right] \stackrel{\simeq}{\longrightarrow} \Sigma^{\infty} B O_{2+}\left[\frac{1}{2}\right]
$$


is an equivalence. There is a zig-zag

$$
\mathrm{BO}_{2} \longrightarrow \mathrm{BSO}_{3} \longleftarrow \mathrm{BSU}_{2}=\mathbb{H} \mathbb{P}^{\infty}
$$

of maps that induce homology equivalences with $\mathbb{Z}\left[\frac{1}{2}\right]$-coefficients, so a zig-zag of homotopy-equivalences after $\mathbb{Z}\left[\frac{1}{2}\right]$-localisation. Thus

$$
\Omega^{\infty} \mathbf{M T O}(2)\left[\frac{1}{2}\right] \simeq Q\left(\mathbb{H} \mathbb{P}_{+}^{\infty}\right)\left[\frac{1}{2}\right]
$$

and on homology $H_{*}\left(\Omega_{0}^{\infty} \mathbf{M T O}(2) ; \mathbb{Z}\left[\frac{1}{2}\right]\right) \cong H_{*}\left(Q_{0}\left(\mathbb{H} \mathbb{P}_{+}^{\infty}\right) ; \mathbb{Z}\left[\frac{1}{2}\right]\right)$ as Hopf algebras. The homology of $Q_{0}\left(\mathbb{H} \mathbb{P}_{+}^{\infty}\right)$ can be completely calculated integrally by piecing together its $\mathbb{F}_{p}$ homologies, as its Bockstein spectral sequence is a functor of that of $\mathbb{H} \mathbb{P}^{\infty}$. This determines the integral homology of $\Omega_{0}^{\infty} \mathbf{M T O}(2)$ except for its 2torsion. By Theorem B the map $\Omega_{0}^{\infty} \mathbf{M T O}(2) \longrightarrow Q_{0}\left(\mathrm{BO}_{2+}\right)$ gives an injection on the $E_{1}$ pages of their Bockstein spectral sequences, but it is not clear what happens on subsequent pages.

In the range $* \leq 6$ only the primes 2 and 3 contribute. The Bockstein spectral sequence for $Q_{0}\left(\mathbb{H} \mathbb{P}_{+}^{\infty}\right)$ at the prime 3 collapses at the $E_{2}$ page, so the only odd primary contribution is a $\mathbb{Z} / 3$ in degree 3 . In this range one can deduce the Bockstein spectral sequence for $\Omega_{0}^{\infty} \mathbf{M T O}(2)$ at the prime 2, as the map above still gives an injection on the $E_{2}$ page, and it collapses at the $E_{3}$ page. The only unusual contribution is a $\mathbb{Z} / 4$ in degree 3.

\section{A polynomial family in the mod 2 cohomology of the stable nonorientable mapping class group}

Let $F \longrightarrow E \stackrel{\pi}{\longrightarrow} B$ be a bundle of surfaces. We can define mod 2 characteristic classes as follows. There is an associated first real cohomology bundle $V$, with fibre $H^{1}\left(F_{b} ; \mathbb{R}\right)$ over the point $b \in B$. Define

$$
\chi_{i}(E):=w_{i}(V) \in H^{i}\left(B ; \mathbb{F}_{2}\right)
$$

the $i$-th Stiefel-Whitney class of this vector bundle. The main result is that in the stable nonorientable mapping class group these account for one indecomposable generator in each dimension, and are stably independent.

Theorem 6.1 In the nonorientable mapping class groups, the map

$$
\mathbb{F}_{2}\left[\chi_{1}, \chi_{2}, \chi_{3}, \ldots\right] \longrightarrow H^{*}\left(B \mathcal{N}_{g} ; \mathbb{F}_{2}\right)
$$

is an injection in the stable range $* \leq(g-3) / 4$. 
The theorem of Korkmaz given in Section 2.2 implies that $H^{1}\left(B \mathcal{N}_{g} ; \mathbb{F}_{2}\right) \cong \mathbb{F}_{2}$ for $g \geq 7$, so $\chi_{1}$ is the generator. This gives another interpretation of $\chi_{1}$ : it is the obstruction to reducing the structure group of a bundle of nonorientable surfaces to the index 2 subgroup of the mapping class group generated by Dehn twists.

The proof of the theorem is somewhat indirect. There is a class $1 \in K O^{0}(E)$ representing the trivial 1-dimensional bundle, and applying the Becker-Gottlieb transfer in real K-theory we obtain a virtual bundle $\operatorname{trf}_{\pi}^{*}(1) \in K O^{0}(B)$. An application of the Atiyah-Singer index theorem for families due to Becker and Schultz implies the following theorem, which gives a homotopy-theoretic characterisation of the virtual bundle $V$.

Theorem 6.2 Suppose the surface bundle $E \stackrel{\pi}{\longrightarrow} B$ is smooth and the fibres are compact, connected and nonorientable. Then the K-theory class $\operatorname{trf}_{\pi}^{*}(1)$ coincides with $1-V$ in $K O^{0}(B)$. In particular $\chi_{i}(E)=w_{i}\left(-\operatorname{trf}_{\pi}^{*}(1)\right)$ in $H^{i}\left(B ; \mathbb{F}_{2}\right)$.

Proof By $\left[2\right.$, Theorem 6.1], $\operatorname{trf}_{\pi}^{*}(1)=\sum_{i}(-1)^{i}\left[H^{i}\left(F_{b} ; \mathbb{R}\right)\right]$. In our situation this sum is $1-V$ as $\left[H^{0}\left(F_{b} ; \mathbb{R}\right)\right]$ is the constant rank 1 vector bundle, $\left[H^{1}\left(F_{b} ; \mathbb{R}\right)\right]$ is the vector bundle $V$, and the higher terms are 0 as the fibres $F_{b}$ are nonorientable surfaces.

The virtual bundle $-\operatorname{trf}_{\pi}^{*}(1)$ is classified by

$$
B \stackrel{\operatorname{trf}}{\longrightarrow} Q\left(E_{+}\right) \stackrel{\text { collapse }}{\longrightarrow} Q\left(S^{0}\right) \stackrel{Q(i)}{\longrightarrow} \mathbb{Z} \times B O \stackrel{\chi}{\longrightarrow} \mathbb{Z} \times B O
$$

where $Q(i)$ is the extension to the free infinite loop space of the inclusion $i$ of $S^{0}$ to the 0 and 1 components of $\mathbb{Z} \times B O$, and $\chi$ is the inversion map on $\mathbb{Z} \times B O$. The discussion in Section 2.4 implies that the above composition is homotopic to

$$
B \stackrel{\alpha_{E}}{\longrightarrow} \Omega^{\infty} \mathbf{M T O}(2) \stackrel{\omega}{\longrightarrow} Q\left(B O_{2+}\right) \stackrel{\text { collapse }}{\longrightarrow} Q\left(S^{0}\right) \stackrel{Q(i)}{\longrightarrow} \mathbb{Z} \times B O \stackrel{\chi}{\longrightarrow} \mathbb{Z} \times B O
$$

as the collapse map $E \longrightarrow *$ can be taken to factor through the map $E \longrightarrow \mathrm{BO}_{2}$ classifying the vertical tangent bundle. Define $\bar{\chi}_{i} \in H^{i}\left(\Omega_{0}^{\infty} \mathbf{M T O}(2) ; \mathbb{F}_{2}\right)$ to be the pullback of the $i$-th Stiefel-Whitney class $w_{i}$ by the composition. We call $\bar{\chi}_{i}$ the universal $\chi_{i}$ class, as by the previous theorem $\alpha_{E}^{*}\left(\bar{\chi}_{i}\right)=\chi_{i}(E) \in H^{i}\left(B ; \mathbb{F}_{2}\right)$ for any smooth, nonorientable surface bundle with compact fibres.

Lemma 6.3 The composition $Q(i) \circ$ collapse $\circ \omega: \Omega_{0}^{\infty} \mathbf{M T O}(2) \longrightarrow B O$ is injective on $\mathbb{F}_{2}$-cohomology. The same is true after applying the inversion map $\chi$, as it is a homotopy equivalence. 
Proof It is enough to show that the map is surjective on $\mathbb{F}_{2}$-homology, and to do this it is enough to show that the composition

$$
\Omega^{\infty} \mathbf{M T O}(2) \stackrel{\omega}{\longrightarrow} Q\left(B_{2+}\right) \stackrel{\text { collapse }}{\longrightarrow} Q\left(S^{0}\right) \stackrel{Q(i)}{\longrightarrow} \mathbb{Z} \times B O
$$

without restriction to 0 components is surjective on $\mathbb{F}_{2}$-homology. The action of the Dyer-Lashof algebra on the $\mathbb{F}_{2}$-homology of $\mathbb{Z} \times B O$ has been computed by Priddy [15], and it is generated over the Dyer-Lashof algebra (and Pontrjagin product) by the class $x_{0} \in H_{0}\left(\mathbb{Z} \times B O ; \mathbb{F}_{2}\right)$ representing the component $\{1\} \times B O$. As the composition is a map of infinite loop spaces, it is enough to show that the class $x_{0}$ is in the image.

The composition

$$
\pi_{0}(B) \stackrel{\pi_{0}\left(\alpha_{E}\right)}{\longrightarrow} \pi_{0}\left(\Omega^{\infty} \mathbf{M T O}(2)\right) \cong \mathbb{Z} \longrightarrow \pi_{0}\left(Q\left(S^{0}\right)\right) \cong \mathbb{Z}
$$

for a surface bundle $E \stackrel{\pi}{\rightarrow} B$ picks out the Euler characteristic of the fibre. In particular, any bundle with fibre $\mathbb{R} \mathbb{P}^{2}$ lands in the 1 component, and so the top composition is an isomorphism on $\pi_{0}$. In particular, the class $x_{0}$ is in the image.

The lemma implies that

$$
\mathbb{F}_{2}\left[\bar{\chi}_{1}, \bar{\chi}_{2}, \bar{\chi}_{3}, \ldots\right] \longrightarrow H^{*}\left(\Omega_{0}^{\infty} \mathbf{M T O}(2) ; \mathbb{F}_{2}\right)
$$

is injective, because the Stiefel-Whitney classes are algebraically independent in $H^{*}\left(B O ; \mathbb{F}_{2}\right)$. Theorem 6.1 follows by the homology stability of Section 2.1.

\section{References}

[1] J C Becker, D H Gottlieb, The transfer map and fiber bundles, Topology 14 (1975) 1-12 MR0377873

[2] J C Becker, R E Schultz, The real semicharacteristic of a fibered manifold, Quart. J. Math. Oxford Ser. (2) 33 (1982) 385-403 MR679811

[3] G Brumfiel, I Madsen, Evaluation of the transfer and the universal surgery classes, Invent. Math. 32 (1976) 133-169 MR0413099

[4] F R Cohen, T J Lada, J P May, The homology of iterated loop spaces, Lecture Notes in Math. 533, Springer, Berlin (1976) MR0436146

[5] W G Dwyer, Exotic convergence of the Eilenberg-Moore spectral sequence, Illinois J. Math. 19 (1975) 607-617 MR0383409

[6] J Ebert, O Randal-Williams, On the divisibility of characteristic classes of nonoriented surface bundles, to appear in Topology Appl. (2008) arXiv:0707.0198 
[7] S Galatius, Mod p homology of the stable mapping class group, Topology 43 (2004) 1105-1132 MR2079997

[8] S Galatius, I Madsen, U Tillmann, M Weiss, The homotopy type of the cobordism category arXiv:math/0605249

[9] M Korkmaz, First homology group of mapping class groups of nonorientable surfaces, Math. Proc. Cambridge Philos. Soc. 123 (1998) 487-499 MR1607985

[10] T Kudo, S Araki, Topology of $H_{n}$-spaces and $H$-squaring operations, Mem. Fac. Sci. Kyūsyū Univ. Ser. A. 10 (1956) 85-120 MR0087948

[11] I Madsen, U Tillmann, The stable mapping class group and $Q\left(\mathbb{C} P_{+}^{\infty}\right)$, Invent. Math. 145 (2001) 509-544 MR1856399

[12] I Madsen, M Weiss, The stable moduli space of Riemann surfaces: Mumford's conjecture, Ann. of Math. (2) 165 (2007) 843-941 MR2335797

[13] B M Mann, E Y Miller, H R Miller, $S^{1}$-equivariant function spaces and characteristic classes, Trans. Amer. Math. Soc. 295 (1986) 233-256 MR831198

[14] J W Milnor, J C Moore, On the structure of Hopf algebras, Ann. of Math. (2) 81 (1965) 211-264 MR0174052

[15] S Priddy, Dyer-Lashof operations for the classifying spaces of certain matrix groups, Quart. J. Math. Oxford Ser. (2) 26 (1975) 179-193 MR0375309

[16] N Wahl, Homological stability for the mapping class groups of non-orientable surfaces, Invent. Math. 171 (2008) 389-424 MR2367024

Mathematical Institute

24-29 St Giles', Oxford, OX1 3LB, UK

randal-w@maths.ox.ac.uk

http://people.maths.ox.ac.uk/ randal-w/

Received: 2 April 2008 Revised: 11 September 2008 